\title{
Two New Species of Taeniogyrus and Scoliorhapis (Echinodermata: Holothuroidea: Apodida: Chiridotidae: Taeniogyrinae) from Wakayama and Okinawa, Japan
}

\author{
YUSUKE YAMANA $^{1, *} \&$ HAYATO TANAKA ${ }^{2}$ \\ ${ }^{1}$ Wakayama Prefectural Museum of Natural History, Funo 370-1, Kainan, Wakayama 642-0001, Japan \\ ${ }^{2}$ Research Center for Marine Education, Ocean Alliance, The University of Tokyo, Hongo 7-3-1, Bunkyo-ku, Tokyo 113-0033, \\ Japan
}

Received 27 September 2016; Accepted 4 January 2017 Responsible Editor: Shigeaki Kojima

\begin{abstract}
Two new taeniogyrinid sea cucumbers collected in the intertidal zone of southern Japan, Taeniogyrus verruculosus sp. nov. and Scoliorhapis sesokoensis sp. nov. are described. Both species possess a small body size (approximately 30-40 mm), light body color (translucent white in preserved specimens), ten tentacles, and numerous verrucous surfaces on the body, within which small (mostly less than $70 \mu \mathrm{m}$ in length) sigmoid-hook ossicles are present. The two new species have a very similar external appearance to each other, however they do differ in the number of tentacle digits: T. verruculosus possesses up to eight digits, whereas $S$. sesokoensis possesses up to six. In both species, wheel papillae are not present. T. verruculosus possesses only a few small (mostly less than $30 \mu \mathrm{m}$ in diameter) wheel ossicles in the anterior dorsal skin, which are not present in S. sesokoensis. Additionally, the DNA barcode sequences of two new species were determined and are available in the DDBJ/NCBI/GenBank databases under the accession numbers.
\end{abstract}

Key words: Chiridotidae, DNA barcode, Scoliorhapis sesokoensis sp. nov., Taeniogyrus verruculosus sp. nov.

\section{Introduction}

Recently, many intertidal sea cucumbers of the order Apodida [or Synaptida; see Smirnov (2012) regarding their higher-level classification] have been collected in southern Japan, including some species that appear distinct from all others reported in Japan. In this report, two of them are described and compared with their congeners using careful survey of the literature. We determine these two species to be a new discovery.

Regarding their ossicle morphologies, one species possesses sigmoid-hook osscles, and the other one possesses sigmoid-hooks as well as wheel ossicles in the outer skin. The former feature is identical to the genus Scoliorhapis Clark, 1946, and the latter feature is identical to the genus Taeniogyrus Semper, 1868. Although our two new species are different in ossicle morphology, they possess many similar features in the external and internal morphologies. Therefore, we also determined the DNA sequence data of the mitochondrial cytochrome c oxidase subunit 1 (COl)

\footnotetext{
*Corresponding author: Yusuke Yamana; E-mail, yamanamako@gmail. com
}

gene for each new species in order to aid future efforts in species identification.

\section{Materials and Methods}

Animals were collected from the lower intertidal zone of sandy-gravel shores at the following sites in southern Japan: (MOT) Motoshima Islet, Mera, Tanabe, Wakayama $\left(33^{\circ} 43^{\prime} 56.0^{\prime \prime} \mathrm{N}, 135^{\circ} 20^{\prime} 58.0^{\prime \prime} \mathrm{E}\right), 17$ June 2015, 7 July 2016 (coll. Y. Yamana); (SES) Sesoko Island, Motobu, Okinawa $\left(26^{\circ} 39^{\prime} 02.6^{\prime \prime} \mathrm{N}, 127^{\circ} 51^{\prime} 24.1^{\prime \prime} \mathrm{E}\right), 7$ October 2014 (coll. H. Tanaka), 10-12 February 2016 (coll. Y. Yamana).

Large specimens were collected by hand net, from sediments approximately $10 \mathrm{~cm}$ below the sea floor. Small specimens were collected using the following method: several scoops of sediment were taken approximately $30 \mathrm{~cm}$ below the sea floor, and were quickly washed with seawater in a bucket to suspend interstitial animals before the water was filtered through a $40-100 \mu \mathrm{m}$ mesh sieve. The specimens from these filtered samples were sorted live under a binocular dissecting microscope (Nikon SMZ). Sorted specimens were fixed and preserved in $80 \%$ ethanol. Some specimens were fixed after anaesthetization in a 
menthol solution $(0.37 \mathrm{~g}$ of menthol dissolved into $100 \mathrm{~mL}$ of $99 \%$ ethanol, this then being diluted to $3.0 \%$ with seawater) for $30 \mathrm{~min}$.

All specimens in the type series were dissected, with the tentacles, Polian vesicles, ciliated funnels, and calcareous rings then examined under a binocular dissecting microscope (Nikon SMZ); drawings of the tentacles and calcareous rings were made with a camera lucida. Ossicles from four regions of the body wall (dorsal anterior, dorsal posterior, ventral anterior, and ventral posterior) and the lateral tentacles were characterized. To extract ossicles, small pieces of tissue were isolated using sharpened ophthalmologic scissors under the dissecting microscope, and dissolved using sodium hypochlorite solution ( $\mathrm{NaClO}$, $5 \%$ ). Ossicles extracted from all five parts were mounted on glass slides in polyester resin (Showadenko RIGOLAC) and characterized under a compound microscope (Nikon Optiphot). Several ossicles from the tentacles and the anterior dorsal body-wall were rinsed with deionized water, dehydrated in $99 \%$ ethanol, mounted on aluminium stubs using conductive tape (Nisshin NEM Tape), dried at room temperature, and characterized using a scanning electron microscope (SEM) (Nippondenshi JEOL JSM-6480LV).

The ossicles were classified into 'wheels' (i.e. wheel-like ossicles with spokes and a series of teeth on the inner margin of the rim), 'sigmoid-hooks' (fishhook-like ossicles, with an open pointed end and closed blunt end, with the point of the hook turned outwards), and 'rods' (rod-like ossicles, slightly curved, sometimes distally branching or spreading). Measurements of 11-21 ossicles were taken to the nearest micrometer for every tissue sample. To compare these measurements among individuals and among parts of the body, Kruskal-Wallis tests were performed. The dissected specimens, glass slides, and SEM material were deposited in the Invertebrate Collection (INV) of the Wakayama Prefectural Museum of Natural History (WMNH) in Kainan, Wakayama, Japan.

DNA extractions from small pieces of ethanol-preserved tissue were performed using the DNeasy Blood and Tissue Kit (Qiagen, USA) following the manufacturer's protocol. Partial sequences of the mitochondrial cytochrome $c$ oxidase subunit $1(\mathrm{COl})$ gene were PCR-amplified using the following primer set: forward (LCO1490; Folmer et al. 1994) and reverse (COle-R; Arndt et al. 1996). The $25 \mu \mathrm{L}$ reaction contained $0.125 \mu \mathrm{L}$ of TaKaRa Ex Taq HS (TAKARA BIO Inc., Japan), $2.5 \mu \mathrm{L}$ of $10 \times E x$ Taq buffer, $2 \mu \mathrm{L}$ of dNTP mix, $1 \mu \mathrm{L}$ of each primer (5 pmol each), $1 \mu \mathrm{L}$ of template DNA, and $17.375 \mu \mathrm{L}$ of sterilized distilled water. The PCR protocol included an initial denaturation step at $95^{\circ} \mathrm{C}$ for $2 \mathrm{~min}$, followed by 40 cycles of denaturation at $98^{\circ} \mathrm{C}$ for $10 \mathrm{~s}$, annealing at $45^{\circ} \mathrm{C}$ for $30 \mathrm{~s}$, and extension at $72^{\circ} \mathrm{C}$ for $1 \mathrm{~min}$, and a final extension at $72^{\circ} \mathrm{C}$ for $5 \mathrm{~min}$. Quantity and length of the PCR products were checked by $1 \%$ agarose S (Nippon Gene, Japan) gel electrophoresis, stained with ethidium bromide. The products were purified for sequencing using a FastGene Gel/PCR Extraction
Kit (NIPPON Genetics Co, Ltd, Japan), according to the manufacturer's protocol. Sequencing (of both the forward and reverse reads) was performed by the Macrogen Japan Corp. (Tokyo) with the same primers used for PCR amplification. A homology search of $\mathrm{COl}$ sequences was performed by BLAST (Altschul et al. 1990, 1997) with the Discontiguous MegaBLAST program from the National Center for Biotechnology Information (NCBI, http://blast. ncbi.nlm.nih.gov/Blast.cgi).

The evolutionary distances of the nucleotide sequences were estimated with MEGA6 (Tamura et al. 2013) using Kimura's two parameter model (Kimura 1980). Standard error estimates were obtained by a bootstrap procedure (1000 replicates).

\section{Taxonomy}

\author{
Order Apodida Brandt, 1835 (sensu Östergren, 1907) \\ Family Chiridotidae Östergren, 1898 \\ Subfamily Taeniogyrinae Smirnov, 1998 \\ Genus Taeniogyrus Semper, 1868
}

Taeniogyrus verruculosus $\mathrm{sp}$. nov. (Figs 1A, B, 2A, C, 3A, B, C)

Material examined Holotype: WMNH-INV-2015-154 (MOT, 17 June 2015) (length $23.9 \mathrm{~mm}$, width $3.0 \mathrm{~mm}$ ). Paratypes: WMNH-INV-2016-205 (MOT, 7 July 2016) (length $27.8 \mathrm{~mm}$, width $2.7 \mathrm{~mm}$ ); WMNH-INV-2016-206 (MOT, 7 July 2016) (anterior half only: length $8.2 \mathrm{~mm}$, width $3.2 \mathrm{~mm}$ ).

Other material: 1 specimen, WMNH-INV-2016-207 (MOT, 7 July 2016) (posterior half only: length $6.4 \mathrm{~mm}$, width $1.9 \mathrm{~mm}$ ).

Description Body small, anesthetized length of largest specimen (WMNH-INV-2016-205) $27.8 \mathrm{~mm}$ (after preservation), cylindrical, slightly tapered toward posterior end (Table 1). Body color, translucent pale yellowish white to pale orange in living specimens (Fig. 1A), and translucent white in preserved specimens (Fig. 1B). Numerous verrucous surfaces present in anterior body skin, sparse posterior. Mouth anterior; anus posterior. Oral disc slightly inclined toward ventral side.

Verrucous surfaces, high, hollow, with a blunt conical form, dense in inter-radius, sparse in radius. Color of verrucous surfaces same as adjacent body skin (preserved and living specimens). Size and shape of verrucous surfaces almost uniform, height approximately $0.2-0.4 \mathrm{~mm}$ and width of basal part approximately $0.2-0.3 \mathrm{~mm}$ in largest specimen (paratype, WMNH-INV-2016-205), becoming gradually smaller as body size decreases.

Calcareous ring composed of five radial and five interradial plates, these all being centrally contracting, and distally spreading ends (Fig. 2A). Thin retractor muscles, combined with longitudinal muscles by mesentery. Ten tentacles non-retractile, each with up to four pairs of digits 
Table 1. Summary of measurements, and counts for type specimens of Taeniogyrus verruculosus sp. nov. and Scoliorhapis sesokoensis sp. nov.

\begin{tabular}{|c|c|c|c|c|c|c|c|c|c|c|c|}
\hline \multirow{3}{*}{ Registration $^{\mathrm{a}}$} & \multirow{3}{*}{$\begin{array}{l}\text { Type } \\
\text { status }\end{array}$} & \multirow{3}{*}{ Locality $^{\mathrm{b}}$} & \multirow{3}{*}{ Date } & \multirow{3}{*}{$\begin{array}{l}\text { Body } \\
\text { state }\end{array}$} & \multirow{2}{*}{\multicolumn{2}{|c|}{$\begin{array}{l}\text { Body size } \\
(\mathrm{mm})\end{array}$}} & \multicolumn{4}{|c|}{ Counts } & \multirow{3}{*}{$\begin{array}{l}\text { Gonad } \\
\text { state }\end{array}$} \\
\hline & & & & & & & \multirow{2}{*}{$\begin{array}{l}\text { Ten- } \\
\text { tacles }\end{array}$} & \multirow{2}{*}{$\begin{array}{l}\text { Tentacle } \\
\text { digits }\end{array}$} & \multirow{2}{*}{$\begin{array}{l}\text { Madre } \\
\text { porite }\end{array}$} & \multirow{2}{*}{$\begin{array}{l}\text { Polian } \\
\text { vesicles }\end{array}$} & \\
\hline & & & & & Length & Width & & & & & \\
\hline \multicolumn{12}{|c|}{ T. verruculosus sp. nov. } \\
\hline $\begin{array}{l}\text { WMNH- } \\
\text { INV-2015-154 }\end{array}$ & holotype & MOT & 17 June 2015 & anaesthetized & 23.9 & 3.0 & 10 & $5-8$ & 1 & 1 & testis \\
\hline $\begin{array}{l}\text { WMNH- } \\
\text { INV-2016-205 }\end{array}$ & paratype & MOT & 7 July 2016 & anaesthetized & 27.8 & 2.7 & 10 & $6-7$ & 1 & 1 & testis \\
\hline $\begin{array}{l}\text { WMNH- } \\
\text { INV-2016-206 }\end{array}$ & paratype & MOT & 7 July 2016 & anterior half & 8.2 & 3.2 & 10 & $6-7$ & 1 & 1 & ovary \\
\hline \multicolumn{12}{|l|}{ S. sesokoensis sp. nov. } \\
\hline $\begin{array}{l}\text { WMNH- } \\
\text { INV-2015-721 }\end{array}$ & paratype & SES & 7 October 2014 & $\begin{array}{l}\text { unanaesthe- } \\
\text { tized }\end{array}$ & 2.4 & 1.0 & 8 & 4 & 1 & 1 & immature \\
\hline $\begin{array}{l}\text { WMNH- } \\
\text { INV-2015-722 }\end{array}$ & paratype & SES & 7 October 2014 & $\begin{array}{l}\text { unanaesthe- } \\
\text { tized }\end{array}$ & 3.3 & 0.6 & 9 & $3-4$ & 1 & 1 & immature \\
\hline $\begin{array}{l}\text { WMNH- } \\
\text { INV-2016-53 }\end{array}$ & holotype & SES & $\begin{array}{c}10-12 \text { February } \\
2016\end{array}$ & anaesthetized & 36.5 & 3.0 & 10 & 6 & 1 & 1 & testis \\
\hline $\begin{array}{l}\text { WMNH- } \\
\text { INV-2016-54 }\end{array}$ & paratype & SES & $\begin{array}{c}\text { 10-12 February } \\
2016\end{array}$ & anaesthetized & 31.7 & 2.9 & 10 & 6 & 1 & 1 & testis \\
\hline $\begin{array}{l}\text { WMNH- } \\
\text { INV-2016-55 }\end{array}$ & paratype & SES & $\begin{array}{c}10-12 \text { February } \\
2016\end{array}$ & anaesthetized & 14.9 & 1.8 & 10 & $4-6$ & 1 & 1 & immature \\
\hline
\end{tabular}

${ }^{a}$ WMNH-INV: Invertebrate Collection of the Wakayama Prefectural Museum of Natural History

${ }^{\text {b }}$ MOT: Motoshima Islet, Mera, Tanabe, Wakayama, SES: Sesoko Island, Motobu, Okinawa

(sometimes unpaired digit present), tip pair largest; sensory cups absent (Fig. 2C). Color of tentacles translucent white (preserved and living specimens). Polian vesicle single, medioventral, and madreporite single (Table 1). Intestine has no loop. Two long bundles of ovaries or testis present, each with approximately 3-4 tubules, mostly unbranched except in basal part. One row of ciliated funnels situated in inter-radius of anterior to posterior body cavity, in left lateral side, dense in anterior, sparse in posterior. One narrow row of ciliated funnels situated in inter-radius of posterior body cavity, in mid-dorsal side, sparse. Sometimes 5-15 ciliated funnels clustered in a bundle.

Body wall containing sigmoid-hook ossicles and wheel ossicles (Figs 1B, 3B, C, Table 2). Sigmoid-hook ossicles, small, scattered around body surface in both radii and inter-radii, approximately 15-25 hooks arranged in one direction on verrucous surface, similar in appearance to strawberry seeds. Pointed end facing toward tip of verrucous surface, and blunt end facing toward base of verrucous surface. Length of shank, 39-68 $\mu$ m (means of 51-62 $\mu \mathrm{m}$ in different specimens) in anterior dorsal side, $36-66 \mu \mathrm{m}$ (means 53 and $58 \mu \mathrm{m}$ ) in anterior ventral side, $38-71 \mu \mathrm{m}$ (means $48-64 \mu \mathrm{m}$ ) in posterior dorsal side, and 42-64 $\mu \mathrm{m}$ (means 48 and $59 \mu \mathrm{m}$ ) in posterior ventral side. Measurements significantly different among four parts in two complete specimens (WMNH-INV-2015-154 and 2016-205) (Kruskal-Wallis test, $P_{\mathbf{S}}<0.05$ ), and measurements of anterior dorsal and ventral parts significantly dif- ferent among three counted specimens (Kruskal-Wallis test, $P \mathrm{~s}<0.01)$.

Wheel ossicles rounded-hexagonal with six spokes (sometimes five or seven). Inner margin of rim with 6-8 continuous teeth per radiant. Spokes broad, their breadth comprising $18-24 \%$ of wheel ossicle diameter (Fig. 3B). Wheel ossicle aggregations (wheel papillae) absent. Wheel ossicle diameter 25-32 $\mu \mathrm{m}$, only observed in anterior dorsal body-wall. Wheels very sparse, and statistical analysis not applied.

Tentacles containing rod ossicles (Fig. 3A, Table 2). Rod ossicles arced; distally branched. Distal branches curved inward considerably. Sometimes having spinous processes distally, however, less so centrally. Rod ossicle length 39$66 \mu \mathrm{m}$, means of $54-58 \mu \mathrm{m}$ in different specimens, significantly different among three counted specimens (KruskalWallis test, $P<0.01$ ).

Distribution In sandy-gravel sediment in the lower intertidal zone, Motoshima Islet, Wakayama Prefecture, Japan.

Etymology The specific name verruculosus is derived from the Latin word meaning "verrucous".

DNA barcode sequence The sequences of paratypes (WMNH-INV-2016-205, accession number LC203483 and WMNH-INV-2016-206, accession number LC203484) 

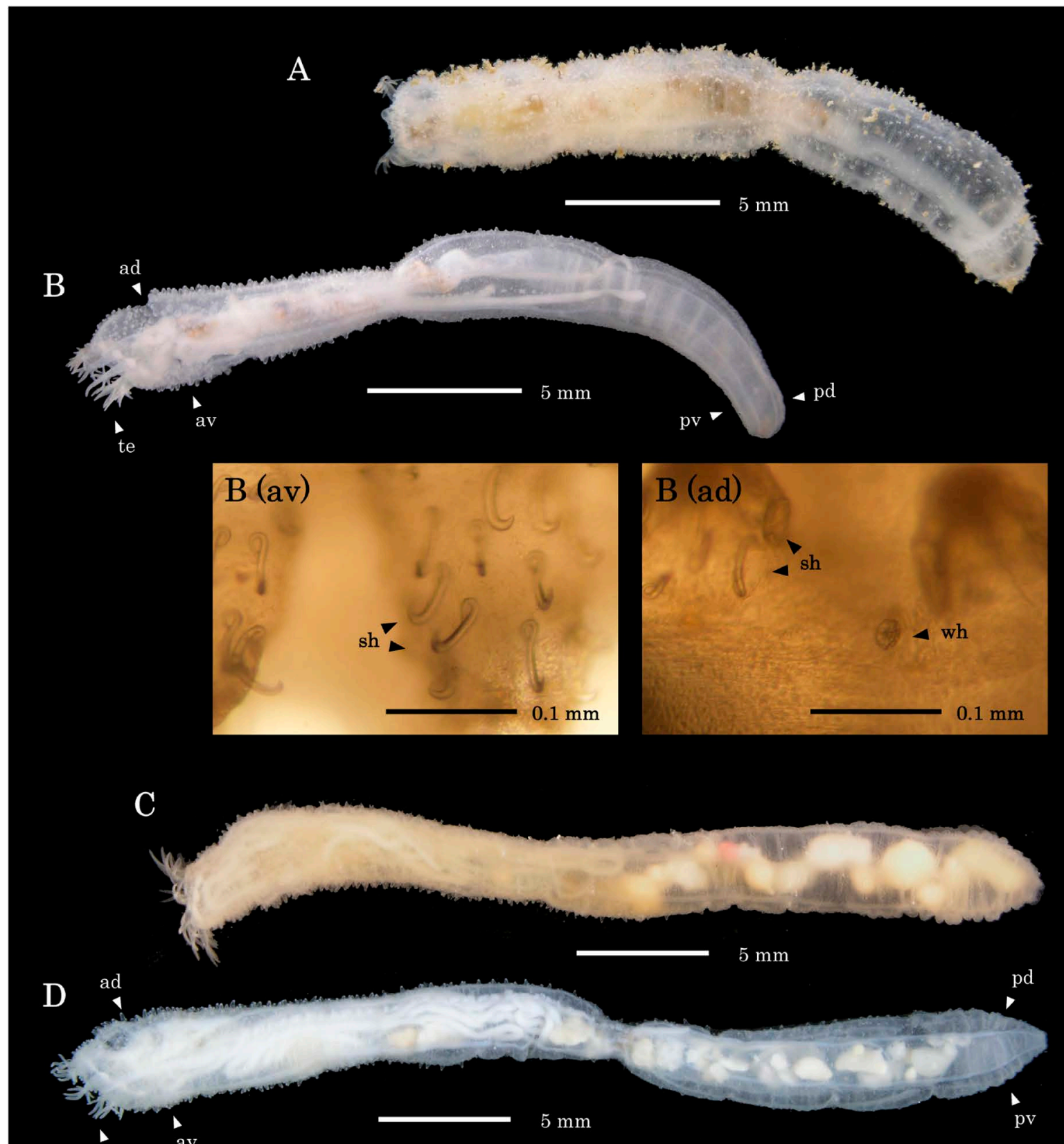

te
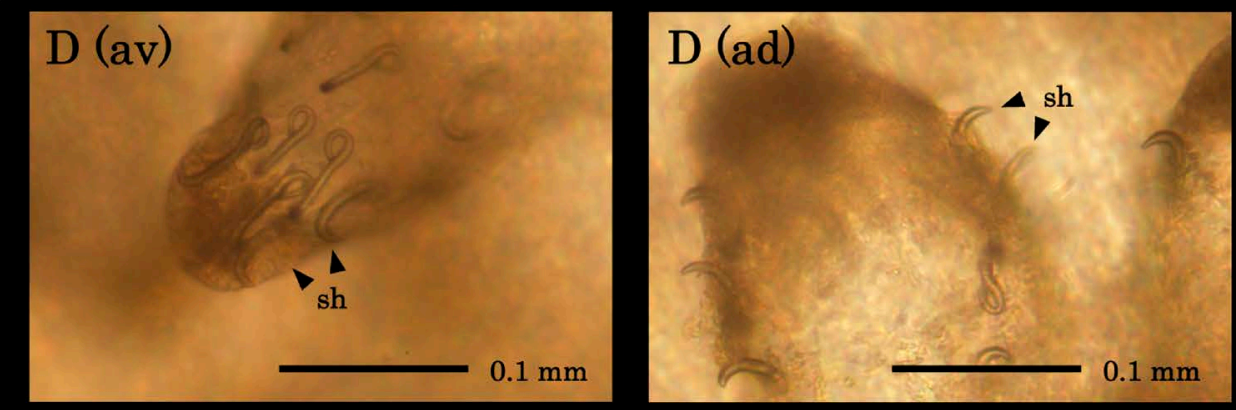

Fig. 1. A and B, lateral view of Taeniogyrus verruculosus sp. nov. A, living state of WMNH-INV-2016-205 (paratype); B, preserved state of WMNH-INV-2015-154 (holotype). B (av) and B (ad), microphotograph of anterior ventral (av) and dorsal (ad) skin containing ossicles of WMNH-INV-2015-154. C and D, lateral view of Scoliorhapis sesokoensis sp. nov. C, living state of WMNH-INV-2016-54 (paratype); D, preserved state of WMNH-INV-2016-53 (holotype). D (av) and D (ad), microphotograph of anterior ventral (av) and dorsal (ad) skin containing ossicles of WMNH-INV-2016-53. Abbreviations: ad, anterior dorsal; av, anterior ventral; pd, posterior dorsal; pv, posterior ventral; sh, sigmoid-hook ossicle; te, tentacle; wh, wheel ossicle. 


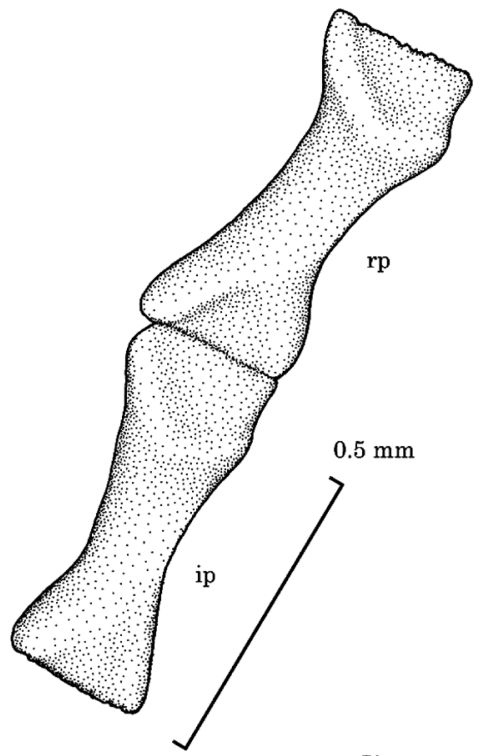

$\mathrm{C}$
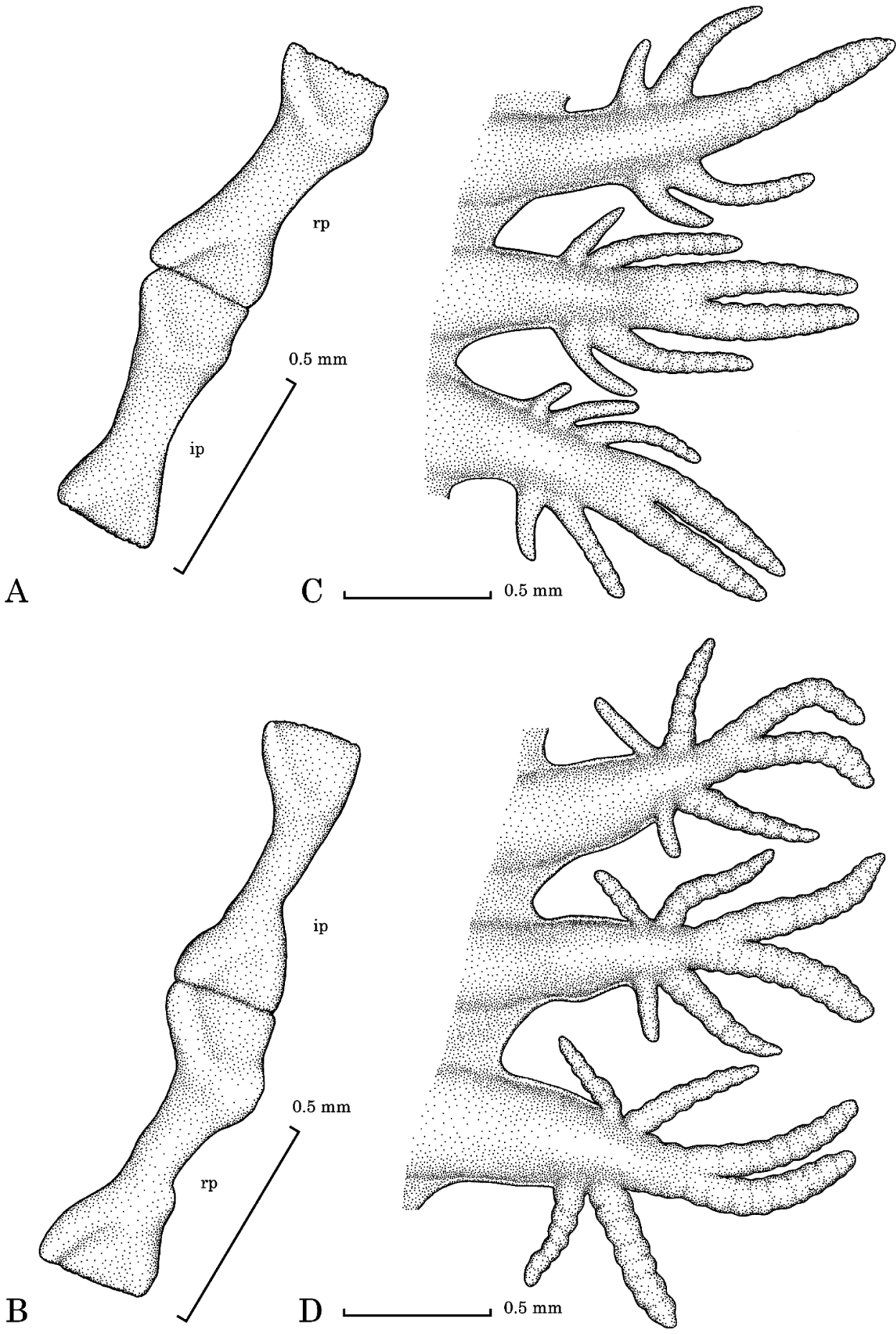

Fig. 2. A and B, one radial and one inter-radial plate each of the calcareous ring on the right side. A, Taeniogyrus verruculosus sp. nov. (holotype, WMNH-INV-2015-154); B, Scoliorhapis sesokoensis sp. nov. (holotype, WMNH-INV-2016-53); viewed from right side with dorsal side above. C and D, tentacles of left side. C, T. verruculosus sp. nov. (holotype, WMNH-INV-2015-154); D, S. sesokoensis sp. nov. (holotype, WMNH-INV-2016-53) ; viewed from right side with dorsal side above. Abbreviations: ip, inter-radial plate; rp, radial plate. 
Table 2. Counts and measurements (Mean \pm sd) of ossicles from body skin for type specimens of Taeniogyrus verruculosus sp. nov.

\begin{tabular}{|c|c|c|c|c|c|c|c|c|c|c|c|}
\hline \multirow[b]{2}{*}{ Registration $^{\mathrm{a}}$} & \multirow[b]{2}{*}{ Body skin } & \multicolumn{3}{|c|}{ Rod } & \multicolumn{3}{|c|}{ Sigmoid-hook } & \multicolumn{4}{|c|}{ Wheel $^{\mathrm{b}}$} \\
\hline & & (n) & $\begin{array}{l}\text { Length } \\
(\mu \mathrm{m})\end{array}$ & $\begin{array}{l}\text { Breadth } \\
(\mu \mathrm{m})\end{array}$ & $(n)$ & $\begin{array}{l}\text { Length } \\
(\mu \mathrm{m})\end{array}$ & $\begin{array}{l}\text { Breadth } \\
\quad(\mu \mathrm{m})\end{array}$ & $(n)$ & $\begin{array}{l}\text { Diameter } \\
\qquad(\mu \mathrm{m})^{\mathrm{c}}\end{array}$ & $\begin{array}{l}\text { Number } \\
\text { of teeth }^{\mathrm{d}}\end{array}$ & $\begin{array}{l}\text { Breadth of } \\
\text { spoke }(\%)^{\mathrm{e}}\end{array}$ \\
\hline \multirow[t]{5}{*}{$\begin{array}{l}\text { WMNH- } \\
\text { INV-2015-154 }\end{array}$} & $\begin{array}{l}\text { Tentacle } \\
\text { (Range) }\end{array}$ & (12) & $\begin{array}{c}55.4 \pm 5.4 \\
(46-62)\end{array}$ & $\begin{array}{c}4.8 \pm 0.5 \\
(4-5)\end{array}$ & (0) & & & $(0)$ & & & \\
\hline & $\begin{array}{l}\text { Anterior dorsal } \\
\text { (Range) }\end{array}$ & (0) & & & (14) & $\begin{array}{c}62.0 \pm 4.5 \\
(53-68)\end{array}$ & $\begin{array}{c}8.1 \pm 0.5 \\
(7-9)\end{array}$ & (4) & $\begin{array}{c}27.0 \pm 1.8 \\
(46-75)\end{array}$ & $\begin{array}{l}6.8 \pm 1.0 \\
(6-8)\end{array}$ & $\begin{array}{c}21.2 \pm 2.2 \\
(19-24)\end{array}$ \\
\hline & $\begin{array}{c}\text { Posterior dorsal } \\
\text { (Range) }\end{array}$ & $(0)$ & & & (19) & $\begin{array}{c}63.7 \pm 4.0 \\
(55-71)\end{array}$ & $\begin{array}{c}8.8 \pm 0.9 \\
(8-11)\end{array}$ & $(0)$ & & & \\
\hline & $\begin{array}{c}\text { Anterior ventral } \\
\text { (Range) }\end{array}$ & (0) & & & (20) & $\begin{array}{c}57.8 \pm 4.9 \\
(47-66)\end{array}$ & $\begin{array}{c}8.3 \pm 0.7 \\
(7-9)\end{array}$ & $(0)$ & & & \\
\hline & $\begin{array}{c}\text { Posterior ventral } \\
\text { (Range) }\end{array}$ & (0) & & & (16) & $\begin{array}{l}59.3 \pm 3.6 \\
(54-64)\end{array}$ & $\begin{array}{c}8.0 \pm 0.7 \\
(7-10)\end{array}$ & $(0)$ & & & \\
\hline \multirow[t]{5}{*}{$\begin{array}{l}\text { WMNH- } \\
\text { INV-2016-205 }\end{array}$} & $\begin{array}{l}\text { Tentacle } \\
\text { (Range) }\end{array}$ & (15) & $\begin{array}{c}53.8 \pm 5.2 \\
(39-60)\end{array}$ & $\begin{array}{c}4.7 \pm 0.8 \\
(4-6)\end{array}$ & $(0)$ & & & $(0)$ & & & \\
\hline & $\begin{array}{c}\text { Anterior dorsal } \\
\text { (Range) }\end{array}$ & $(0)$ & & & (20) & $\begin{array}{c}50.8 \pm 5.8 \\
(39-62)\end{array}$ & $\begin{array}{c}7.0 \pm 0.6 \\
(6-8)\end{array}$ & (1) & $\begin{array}{c}32 \\
(32)\end{array}$ & $\begin{array}{c}7 \\
(7)\end{array}$ & $\begin{array}{l}21.9 \\
(21.9)\end{array}$ \\
\hline & $\begin{array}{c}\text { Posterior dorsal } \\
\text { (Range) }\end{array}$ & $(0)$ & & & (16) & $\begin{array}{c}47.9 \pm 5.3 \\
(38-56)\end{array}$ & $\begin{array}{c}6.1 \pm 0.6 \\
(5-7)\end{array}$ & $(0)$ & & & \\
\hline & $\begin{array}{c}\text { Anterior ventral } \\
\text { (Range) }\end{array}$ & $(0)$ & & & (18) & $\begin{array}{l}52.6 \pm 8.1 \\
(36-63)\end{array}$ & $\begin{array}{c}7.2 \pm 0.9 \\
(5-8)\end{array}$ & $(0)$ & & & \\
\hline & $\begin{array}{c}\text { Posterior ventral } \\
\text { (Range) }\end{array}$ & $(0)$ & & & (16) & $\begin{array}{c}48.4 \pm 5.2 \\
(42-56)\end{array}$ & $\begin{array}{l}6.3 \pm 0.6 \\
(5-7)\end{array}$ & $(0)$ & & & \\
\hline \multirow[t]{3}{*}{$\begin{array}{l}\text { WMNH- } \\
\text { INV-2016-206 }\end{array}$} & $\begin{array}{l}\text { Tentacle } \\
\text { (Range) }\end{array}$ & (15) & $\begin{array}{c}58.2 \pm 6.0 \\
(45-66)\end{array}$ & $\begin{array}{c}5.3 \pm 0.6 \\
(4-6)\end{array}$ & $(0)$ & & & $(0)$ & & & \\
\hline & $\begin{array}{c}\text { Anterior dorsal } \\
\text { (Range) }\end{array}$ & (0) & & & (17) & $\begin{array}{c}58.5 \pm 6.8 \\
(44-67)\end{array}$ & $\begin{array}{c}7.2 \pm 0.5 \\
(6-8)\end{array}$ & (2) & $\begin{array}{c}29.5 \\
(28 \text { and } 31)\end{array}$ & $\begin{array}{c}6.5 \\
(6 \text { and } 7)\end{array}$ & $\begin{array}{c}18.6 \\
(17.9 \text { and 19.4) }\end{array}$ \\
\hline & $\begin{array}{c}\text { Anterior ventral } \\
\text { (Range) }\end{array}$ & (0) & & & (20) & $\begin{array}{c}57.3 \pm 8.8 \\
(38-70)\end{array}$ & $\begin{array}{c}6.9 \pm 0.4 \\
(6-8)\end{array}$ & $(0)$ & & & \\
\hline
\end{tabular}

${ }^{\text {a }}$ WMNH-INV: Invertebrate Collection of the Wakayama Prefectural Museum of Natural History

${ }^{\mathrm{b}}$ Wheels with six spokes were only measured.

${ }^{\mathrm{c}}$ Values were measured at the vertices of a hexagon.

${ }^{\mathrm{d}}$ Values were counted at a radian of a hexagon.

${ }^{\mathrm{e}}$ Values were represented by percentage of breadth of a spoke in a diameter.

\section{Genus Scoliorhapis Clark, 1946}

\section{Scoliorhapis sesokoensis sp. nov.} (Figs 1C, D, 2B, D, 3D, E)

Material examined Holotype: WMNH-INV-2016-53 (SES, 10-12 February 2016) (length $36.5 \mathrm{~mm}$, width $3.0 \mathrm{~mm}$ ). Paratypes: WMNH-INV-2015-721 (SES, 7 October 2014) (length $2.4 \mathrm{~mm}$, width $1.0 \mathrm{~mm}$ ); WMNHINV-2015-722 (SES, 7 October 2014) (length $3.3 \mathrm{~mm}$, width $0.6 \mathrm{~mm}$ ); WMNH-INV-2016-54 (SES, 10-12 February 2016) (length $31.7 \mathrm{~mm}$, width $2.9 \mathrm{~mm}$ ); WMNHINV-2016-55 (SES, 10-12 February 2016) (length $14.9 \mathrm{~mm}$, width $1.8 \mathrm{~mm}$ ).

Other material: 1 specimen, WMNH-INV-2015-720 (SES-1, 7 October 2014); 11 specimens, WMNHINV-2015-1459-1469 (SES, 13 September 2015); 43 speci- mens, WMNH-INV-2016-10-52 (SES, 10-12 February 2016).

Description Body small, anaesthetized length of largest specimen (WMNH-INV-2016-53) $36.5 \mathrm{~mm}$ (after preservation), cylindrical, slightly tapered toward posterior end (Table 1). Body color, translucent pale yellowish white to pale orange in living specimens (Fig. 1C), and translucent white in preserved specimens (Fig. 1D). Numerous verrucous surfaces present in anterior body skin, sparse in posterior body skin. Mouth anterior; anus posterior. Oral disc slightly inclined toward ventral side.

Verrucous surfaces, high, hollow, with a blunt conical form, dense in inter-radius, sparse in radius. Verrucous surfaces undeveloped in small specimens (less than approximately $4 \mathrm{~mm}$ ). Color of verrucous surfaces same as adjacent body skin (preserved and living specimens). Size and shape of verrucous almost uniform, height approxi- 

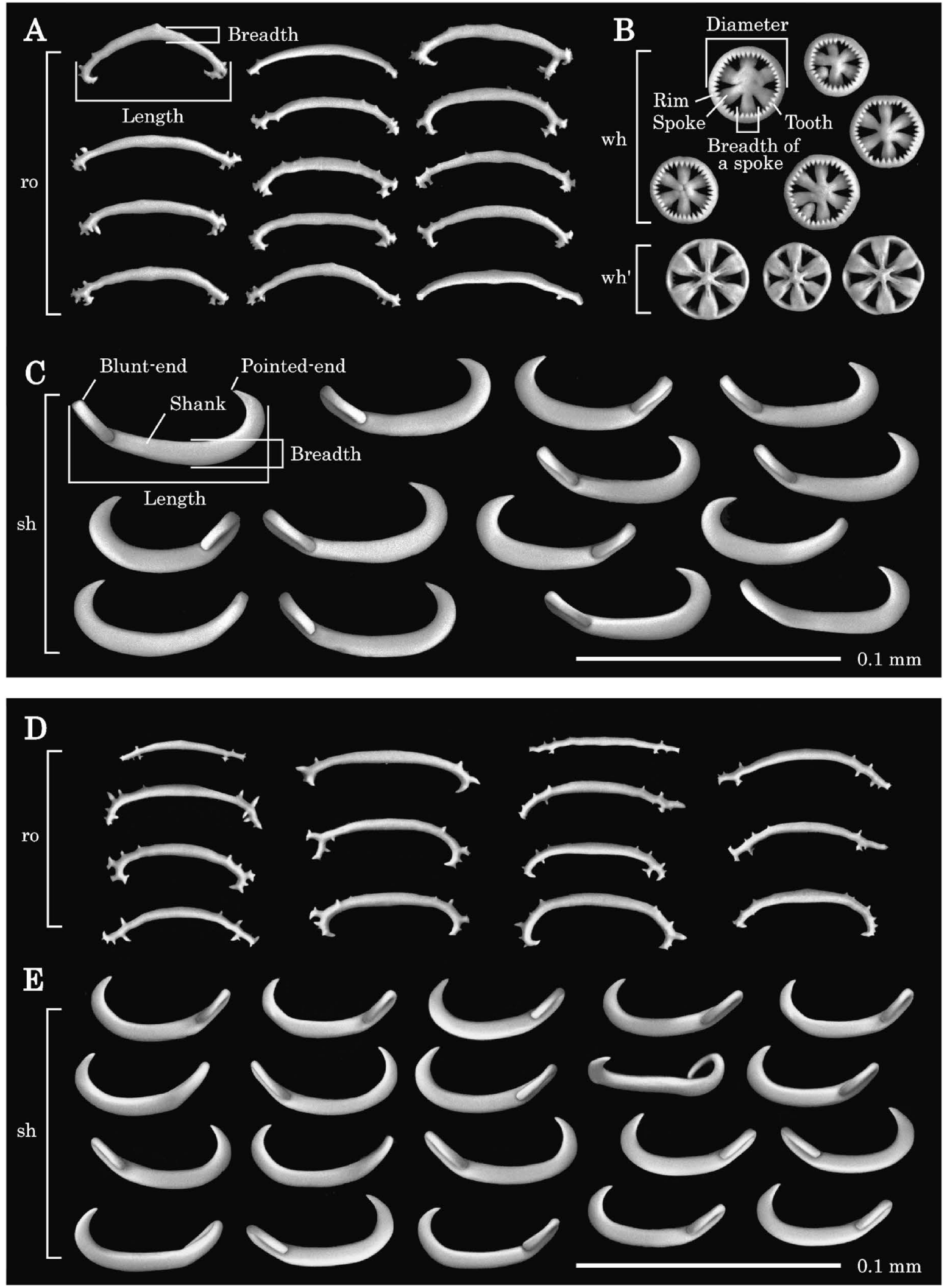

Fig. 3. A-E, SEM image of ossicles. A, rod ossicles from tentacle; B, wheel ossicles; and C, sigmoid-hook ossicles from anterior dorsal body skin of Taeniogyrus verruculosus sp. nov. (holotype, WMNH-INV-2015-154). D, rod ossicles from tentacle; and E, sigmoid-hook ossicles from anterior dorsal body skin of Scoliorhapis sesokoensis sp. nov. (holotype, WMNH-INV-2016-53). Abbreviations: sh, sigmoid-hook ossicles; ro, rod ossicles; wh, wheel ossicles; wh', internal side of wheel ossicles. 
Table 3. Counts and measurements (Mean $\pm \mathrm{sd}$ ) of ossicles from body skin for type specimens of Scoliorhapis sesokoensis sp. nov.

\begin{tabular}{|c|c|c|c|c|c|c|c|c|c|c|c|}
\hline \multirow[b]{2}{*}{ Registration $^{\mathrm{a}}$} & \multirow[b]{2}{*}{ Body skin } & \multicolumn{3}{|c|}{ Rod } & \multicolumn{3}{|c|}{ Sigmoid-hook } & \multicolumn{4}{|c|}{ Wheel } \\
\hline & & $(n)$ & $\begin{array}{l}\text { Length } \\
(\mu \mathrm{m})\end{array}$ & $\begin{array}{l}\text { Breadth } \\
(\mu \mathrm{m})\end{array}$ & (n) & $\begin{array}{l}\text { Length } \\
(\mu \mathrm{m})\end{array}$ & $\begin{array}{l}\text { Breadth } \\
(\mu \mathrm{m})\end{array}$ & $(n)$ & $\begin{array}{l}\text { Diameter } \\
\quad(\mu \mathrm{m})\end{array}$ & $\begin{array}{l}\text { Number } \\
\text { of teeth }\end{array}$ & $\begin{array}{l}\text { Breadth of } \\
\text { spoke }(\%)\end{array}$ \\
\hline \multirow[t]{5}{*}{$\begin{array}{l}\text { WMNH- } \\
\text { INV-2015-721 }\end{array}$} & $\begin{array}{l}\text { Tentacle } \\
\text { (Range) }\end{array}$ & (12) & $\begin{array}{c}43.3 \pm 4.7 \\
(35-54)\end{array}$ & $\begin{array}{c}4.8 \pm 0.8 \\
(4-6)\end{array}$ & $(0)$ & & & $(0)$ & & & \\
\hline & $\begin{array}{l}\text { Anterior dorsal } \\
\text { (Range) }\end{array}$ & (0) & & & (17) & $\begin{array}{r}39.5 \pm 1.8 \\
(37-43)\end{array}$ & $\begin{array}{c}4.9 \pm 0.3 \\
\quad(4-5)\end{array}$ & $(0)$ & & & \\
\hline & $\begin{array}{c}\text { Posterior dorsal } \\
\text { (Range) }\end{array}$ & (0) & & & (17) & $\begin{array}{r}42.2 \pm 2.5 \\
(37-47)\end{array}$ & $\begin{array}{c}5.1 \pm 0.6 \\
(4-6)\end{array}$ & $(0)$ & & & \\
\hline & $\begin{array}{c}\text { Anterior ventral } \\
\text { (Range) }\end{array}$ & (0) & & & (17) & $\begin{array}{r}39.5 \pm 1.8 \\
(36-42)\end{array}$ & $\begin{array}{c}4.8 \pm 0.5 \\
\quad(4-6)\end{array}$ & $(0)$ & & & \\
\hline & $\begin{array}{c}\text { Posterior ventral } \\
\text { (Range) }\end{array}$ & (0) & & & (17) & $\begin{array}{r}41.8 \pm 1.9 \\
(38-45)\end{array}$ & $\begin{array}{l}5.1 \pm 0.2 \\
(5-6)\end{array}$ & $(0)$ & & & \\
\hline \multirow[t]{5}{*}{$\begin{array}{l}\text { WMNH- } \\
\text { INV-2015-722 }\end{array}$} & $\begin{array}{l}\text { Tentacle } \\
\text { (Range) }\end{array}$ & (11) & $\begin{array}{r}49.2 \pm 6.1 \\
(36-57)\end{array}$ & $\begin{array}{c}4.3 \pm 0.6 \\
\quad(3-5)\end{array}$ & $(0)$ & & & $(0)$ & & & \\
\hline & $\begin{array}{l}\text { Anterior dorsal } \\
\text { (Range) }\end{array}$ & (0) & & & (18) & $\begin{array}{r}40.1 \pm 1.8 \\
(36-43)\end{array}$ & $\begin{array}{l}5.3 \pm 0.5 \\
\quad(5-6)\end{array}$ & (0) & & & \\
\hline & $\begin{array}{c}\text { Posterior dorsal } \\
\text { (Range) }\end{array}$ & (0) & & & (18) & $\begin{array}{r}41.3 \pm 1.7 \\
(38-44)\end{array}$ & $\begin{array}{l}5.3 \pm 0.5 \\
\quad(5-6)\end{array}$ & (0) & & & \\
\hline & $\begin{array}{c}\text { Anterior ventral } \\
\text { (Range) }\end{array}$ & (0) & & & (17) & $\begin{array}{r}39.3 \pm 2.2 \\
(35-43)\end{array}$ & $\begin{array}{c}4.9 \pm 0.4 \\
\quad(4-6)\end{array}$ & (0) & & & \\
\hline & $\begin{array}{c}\text { Posterior ventral } \\
\text { (Range) }\end{array}$ & (0) & & & (17) & $\begin{array}{r}41.5 \pm 2.2 \\
(38-44)\end{array}$ & $\begin{array}{c}4.9 \pm 0.6 \\
(4-6)\end{array}$ & $(0)$ & & & \\
\hline \multirow[t]{5}{*}{$\begin{array}{l}\text { WMNH- } \\
\text { INV-2016-53 }\end{array}$} & $\begin{array}{l}\text { Tentacle } \\
\text { (Range) }\end{array}$ & (16) & $\begin{array}{r}61.4 \pm 7.9 \\
(47-72)\end{array}$ & $\begin{array}{l}5.9 \pm 1.0 \\
\quad(4-7)\end{array}$ & $(0)$ & & & $(0)$ & & & \\
\hline & $\begin{array}{l}\text { Anterior dorsal } \\
\text { (Range) }\end{array}$ & (0) & & & (19) & $\begin{array}{r}54.8 \pm 6.3 \\
(41-68)\end{array}$ & $\begin{array}{l}7.3 \pm 0.6 \\
(6-8)\end{array}$ & $(0)$ & & & \\
\hline & $\begin{array}{c}\text { Posterior dorsal } \\
\text { (Range) }\end{array}$ & (0) & & & (16) & $\begin{array}{r}57.6 \pm 2.7 \\
(53-61)\end{array}$ & $\begin{array}{c}7.0 \pm 0.5 \\
(6-8)\end{array}$ & $(0)$ & & & \\
\hline & $\begin{array}{c}\text { Anterior ventral } \\
\text { (Range) }\end{array}$ & (0) & & & (19) & $\begin{array}{r}53.4 \pm 4.4 \\
(46-64)\end{array}$ & $\begin{array}{l}7.2 \pm 0.5 \\
\quad(6-8)\end{array}$ & $(0)$ & & & \\
\hline & $\begin{array}{c}\text { Posterior ventral } \\
\text { (Range) }\end{array}$ & (0) & & & (17) & $\begin{array}{r}52.2 \pm 4.1 \\
(40-56)\end{array}$ & $\begin{array}{l}6.8 \pm 0.6 \\
(6-8)\end{array}$ & $(0)$ & & & \\
\hline \multirow[t]{5}{*}{$\begin{array}{l}\text { WMNH- } \\
\text { INV-2016-54 }\end{array}$} & $\begin{array}{l}\text { Tentacle } \\
\text { (Range) }\end{array}$ & (11) & $\begin{array}{c}57.6 \pm 6.8 \\
(46-66)\end{array}$ & $\begin{array}{l}4.9 \pm 0.5 \\
\quad(4-6)\end{array}$ & $(0)$ & & & (0) & & & \\
\hline & $\begin{array}{l}\text { Anterior dorsal } \\
\text { (Range) }\end{array}$ & (0) & & & $(20)$ & $\begin{array}{r}50.9 \pm 3.6 \\
(43-58)\end{array}$ & $\begin{array}{c}7.0 \pm 0.6 \\
(6-8)\end{array}$ & $(0)$ & & & \\
\hline & $\begin{array}{c}\text { Posterior dorsal } \\
\text { (Range) }\end{array}$ & (0) & & & (17) & $\begin{array}{r}52.8 \pm 2.1 \\
(48-56)\end{array}$ & $\begin{array}{l}6.8 \pm 0.4 \\
(6-7)\end{array}$ & $(0)$ & & & \\
\hline & $\begin{array}{c}\text { Anterior ventral } \\
\text { (Range) }\end{array}$ & (0) & & & $(20)$ & $\begin{array}{r}53.1 \pm 4.1 \\
(47-61)\end{array}$ & $\begin{array}{l}7.2 \pm 0.5 \\
(6-8)\end{array}$ & $(0)$ & & & \\
\hline & $\begin{array}{c}\text { Posterior ventral } \\
\text { (Range) }\end{array}$ & (0) & & & (17) & $\begin{array}{r}52.4 \pm 2.3 \\
(47-55)\end{array}$ & $\begin{array}{l}6.5 \pm 0.6 \\
(5-7)\end{array}$ & $(0)$ & & & \\
\hline \multirow[t]{5}{*}{$\begin{array}{l}\text { WMNH- } \\
\text { INV-2016-55 }\end{array}$} & $\begin{array}{l}\text { Tentacle } \\
\text { (Range) }\end{array}$ & (15) & $\begin{array}{r}54.6 \pm 6.8 \\
(41-65)\end{array}$ & $\begin{array}{l}5.1 \pm 1.0 \\
(4-7)\end{array}$ & $(0)$ & & & $(0)$ & & & \\
\hline & $\begin{array}{l}\text { Anterior dorsal } \\
\quad \text { (Range) }\end{array}$ & $(0)$ & & & (19) & $\begin{array}{r}55.7 \pm 6.2 \\
(45-64)\end{array}$ & $\begin{array}{l}6.5 \pm 0.6 \\
(5-7)\end{array}$ & $(0)$ & & & \\
\hline & $\begin{array}{c}\text { Posterior dorsal } \\
\text { (Range) }\end{array}$ & (0) & & & (20) & $\begin{array}{r}51.5 \pm 5.7 \\
(39-61)\end{array}$ & $\begin{array}{c}6.1 \pm 0.9 \\
(4-7)\end{array}$ & (0) & & & \\
\hline & $\begin{array}{c}\text { Anterior ventral } \\
\text { (Range) }\end{array}$ & (0) & & & $(20)$ & $\begin{array}{r}51.3 \pm 4.2 \\
(44-58)\end{array}$ & $\begin{array}{c}6.5 \pm 0.7 \\
(5-8)\end{array}$ & $(0)$ & & & \\
\hline & $\begin{array}{c}\text { Posterior ventral } \\
\text { (Range) }\end{array}$ & (0) & & & $(20)$ & $\begin{array}{r}54.7 \pm 4.1 \\
(45-60)\end{array}$ & $\begin{array}{l}6.2 \pm 0.6 \\
(5-7)\end{array}$ & $(0)$ & & & \\
\hline
\end{tabular}

${ }^{a}$ WMNH-INV: Invertebrate Collection of the Wakayama Prefectural Museum of Natural History 
mately $0.2-0.4 \mathrm{~mm}$ and width of basal part approximately $0.2-0.3 \mathrm{~mm}$ in largest specimen (holotype, WMNHINV-2016-53), becoming gradually smaller as body size decreases.

Calcareous ring composed of five radial and five interradial plates, these all being centrally contracting, and distally spreading ends (Fig. 2B). Thin retractor muscles, combined with longitudinal muscles by mesentery. Ten tentacles non-retractile, each with up to three pairs of digits (sometimes unpaired digit present), tip pair largest; sensory cups absent (Fig. 2D). Color of tentacles translucent white (preserved and living specimens). Polian vesicle single, medioventral, and madreporite single (Table 1). Intestine has no loop. Two long bundles of ovaries or testis present, each with approximately $2-4$ tubules, mostly unbranched except in basal part. One wide band of ciliated funnels situated in inter-radius of anterior to posterior body cavity, in left lateral side, dense in anterior, sparse in posterior. One narrow band of ciliated funnels situated in inter-radius of posterior body cavity, in mid-dorsal side, sparse. Sometimes 5-15 ciliated funnels clustered in a bundle.

Body wall containing sigmoid-hook ossicles (Figs 1D, 3E, Table 3), small, scattered in body surface in both radii and inter-radii, approximately $15-30$ hooks arranged in one direction on verrucous surfaces similar in appearance to strawberry seeds. Pointed end facing toward tip of verrucous surfaces, and blunt end facing toward base of verrucous surfaces. Length of shank, 36-68 $\mu$ m (means of $39-56 \mu \mathrm{m}$ in different specimens) in anterior dorsal side, 35-64 $\mu \mathrm{m}$ (means 39-53 $\mu \mathrm{m}$ ) in anterior ventral side, $37-61 \mu \mathrm{m}$ (means $39-53 \mu \mathrm{m}$ ) in posterior dorsal side, and 38-60 $\mu \mathrm{m}$ (means $41-55 \mu \mathrm{m}$ ) in posterior ventral side. Measurements mostly significantly different among four parts (Kruskal-Wallis test, $P \mathrm{~s}<0.05$ ), except for one specimen (WMNH-INV-2016-54), and measurements of four parts significantly different among five counted specimens (Kruskal-Wallis test, $P_{\mathbf{s}}<0.05$ ).

Tentacles containing rod ossicles (Fig. 3D, Table 3). Rod ossicles arced; distally branched. Distal branches curved inward considerably. Sometimes having spinous processes distally, however, poorly in centrally. Rod ossicle length 35-72 $\mu \mathrm{m}$, means of $43-61 \mu \mathrm{m}$ in different specimens, significantly different among five counted specimens (Kruskal-Wallis test, $P<0.01)$.

Distribution In sandy-gravel sediment in the lower in- tertidal zone, Sesoko Island, Okinawa Prefecture, Japan.

Etymology The specific name sesokoensis is derived from the name of the type locality of an island, Sesoko.

DNA barcode sequence The sequences of holotype (WMNH-INV-2016-53, accession number LC203485) and paratype (WMNH-INV-2016-55, accession number LC203486).

\section{Evolutionary distance of nucleotide sequences based on CO1 gene between two new species}

The length of the obtained $\mathrm{COI}$ sequences were $819 \mathrm{bp}$ and the alignment of each sequence contained no insertions and deletions. In Taeniogyrus verruculosus sp. nov., two identical sequences were obtained. Base frequency was $\mathrm{A}=18.9 \%, \mathrm{C}=14.9 \%, \mathrm{G}=23.2 \%, \mathrm{~T}=43.0 \%$. In Scoliorhapis sesokoensis sp. nov., two identical sequences are obtained. Base frequency was $\mathrm{A}=21.9 \%, \mathrm{C}=13.9 \%$, $\mathrm{G}=21.7 \%, \mathrm{~T}=42.5 \%$. The evolutionary distance between two new species was $0.11 \pm 0.01$ (Table 4).

\section{Discussion}

The diagnostic characters of Taeniogyrinae Smirnov, 1998 are as follows: 10 or 12 tentacles; body wall ossicles comprise wheels of chiridotid type and sigmoid-hooks or sigmoid-hooks only. To date, the features of sigmoid-hook ossicles has only been observed in the congener of this group, with the following four genera having sigmoidhook ossicles: Sigmodota Studer, 1876; Scoliorhapis; Taeniogyrus; Rowedota O'Loughlin \& VandenSpiegel, 2010. Among them, Sigmodota has 12 tentacles, and Rowedota has wheels with teeth in six discrete groups, therefore they are not agreeable to the present new species, and only two genera Scoliorhapis and Taeniogyrus can be agreeable.

The genus Scoliorhapis Clark, 1946 (q.v.) replaces the rejected genus Scoliodota Clark, 1907 (q.v.), which was originally erected through the description of Scoliodota japonica Clark, 1907, based on the description of Australian specimens of Taeniogyrus japonicus (Marenzeller, 1881, as Chirodota japonica) (q.v.) of Théel's report (Théel 1886). However, Ohshima (1914) reported that T. japonicus had small wheels in very low frequency, and therefore $T$. japonicus was omitted from the genus Scoliodota (and temporarily belonged to the genus Trochodota Ludwig, 1891), and Clark (1921) had abandoned the genus without taking into consideration the Australian specimens. There-

Table 4. Evolutionary distances of CO1 nucleotide sequences between two new species of Taeniogyrus and Scoliorhapis.

\begin{tabular}{lllll}
\hline & 1 & 2 & 3 & 4 \\
\hline 1 T. verruculosus sp. nov. (WMNH-INV-2016-205) & & $0 *$ & $0.01^{*}$ & $0.01^{*}$ \\
2 T. verruculosus sp. nov. (WMNH-INV-2016-206) & 0 & & $0.01^{*}$ & $0.01^{*}$ \\
3 S. sesokoensis sp. nov. (WMNH-INV-2016-53) & 0.11 & 0.11 & $0 *$ \\
4 & S. sesokoensis sp. nov. (WMNH-INV-2016-55) & 0.11 & 0.11 & 0 \\
\hline
\end{tabular}

*Estimated standard error 
fore, Heding had erected Scoliorhapis theelii (Heding, 1928, as Scoliodota théelii) (q.v.) based on the description of these Australian specimens. Heding's genus Scoliodota had been then a monotypic genus, and a junior homonym of Scoliodota Clark, 1907 contained only S. theelii. After that, due to the belief "Scoliodota is a dead synonym of Trochodota”, Clark (1946) replaced Scoliodota with Scoliorhapis. Furthermore, Oguro (1961) had described a new genus Scoliodotella Oguro, 1961, to replace Scoliodota, because he was not aware of Clark's report (1946). Recently, O'Loughlin \& VandenSpiegel (2010) had synonymized Scoliodotella with Scoliorhapis (O'Loughlin \& VandenSpiegel 2010) and had amended diagnosis of Scoliorhapis as follows: taeniogyrinae with 10 or 12 peltato-digitate tentacles, each with up to eight pairs of digits; single polian vesicle; ciliated funnels present; body wall ossicles sigmoid hooks only, hooks scattered or clustered or aligned (clustering of ossicles was rejected as a reliable generic diagnostic character); lacking wheels in body wall; tentacle ossicles bracket-shaped or rods.

The genus Taeniogyrus Semper, 1868 (q.v.) was originally separated from Chirodota Eschscholtz, 1829, defining Taeniogyrus based on the primary features of the wheel ossicles and sigmoid-hook ossicles in the skin of the body: wheel ossicles aggregated and forming papillae, and sigmoid-hook ossicles scattered uniformly. However, aggregations of wheel ossicles have been observed in only a few species besides the type species [e.g. Taeniogyrus cidaridis Ohshima, 1914; T. heterosigmus Heding, 1931 (q.v.)], and not observed in others. Therefore, some Taeniogyrus species that lack wheel papillae were thought to be better classified in the genus Trochodota Ludwig, 1891, which differs from Taeniogyrus primarily in the lack of aggregations of wheel ossicles (Ludwig 1898). Both Taeniogyrus and Trochodota were considered valid for a long time, although it was difficult to draw a definite line of demarcation between them. Recently, O'Loughlin and VandenSpiegel (2010) revised them and concluded that Trochodota studeri (Theel, 1886), the type species of Trochodota, is a junior synonym of Holothuria (Fistularia) purpurea Lesson, 1830. Furthermore, they placed $H$. (F.) purpurea into Taeniogyrus, as T. purpureus (Lesson, 1830). At the same time, O'Loughlin \& VandenSpiegel (2010) had amended diagnosis of Taeniogyrus as follows: taeniogyrinae with 10 peltato-digitate tentacles, each with up to eight pairs of digits, terminal pair longest; body wall ossicles sigmoid hooks and wheels; teeth on the inner rim of wheels in continuous series; wheels and hooks variably grouped or scattered in body wall (wheels aggregated in body wall, "wheel papilla" were rejected as a reliable generic diagnostic character); no miliary granules; 1-12 polian vesicles; ciliated funnels present.

The diagnosis of Scoliorhapis and Taeniogyrus can apply to each one of the present new species: S. sesokoensis which lacks wheels (an observation for more than 50 specimens, including young and adult) and T. verruculosus which has wheels. Presently, Scoliorhapis consists of five species (Paulay 2015) and Taeniogyrus consists of 26 species (Paulay 2013). Presently, two species of Scoliorhapis and four species of Taeniogyrus have been reported in Japan: S. lindbergi (D'yakonov in D'yakonov, Baranova, \& Savael'eva, 1958) from Hokkaido (Oguro 1961, as Scoliodotella uchidai Oguro, 1961); S. dianthus Solis-Marin, Komatsu, Soliman, Uchida, Shimotani, Nozaki, 2014 from the Sea of Japan (Solis-Marin et al. 2014); T. cidaridis Ohshima, 1915 from Goto Islands, off Sagami Bay, and Uraga Strait (Ohshima 1914, 1915); T. misakiensis (Ohshima, 1919) from off. Sagami Bay (Ohshima 1914, 1919); T. japonicus (Marenzeller, 1881) from Sagami Bay (Marenzeller 1881; Ohshima 1914) and from several regions of middle and southern Japan (Utinomi 1965; Imaoka 1995); and T. roseus (Ohshima, 1914) from Okinawa (Ohshima 1914). In the present observation, none of the congeners were agreeable to the two new species.

For the "clustering of ossicles" in both the genera, a kind of clustering of sigmoid-hooks had been reported in the nominal three species: Scoliorhapis theelii, Taeniogyrus japonicus and T. dendyi (Mortensen, 1925), as "hook papilla”, in which sigmoid-hooks were arranged radially in a single circle. The length and the number of sigmoid-hooks are $100-140 \mu \mathrm{m}, 63-120 \mu \mathrm{m}$, and $80-140 \mu \mathrm{m}$, numbering approximately 10 but up to 16 , and 3-6 in each papillae of $S$. theelii, $T$. japonicus and $T$. dendyi, respectively (Theel 1886; Ohshima 1914; Mortensen 1925; Heding 1928). In the two new species, however, 15-30 very small sigmoidhooks (mostly less than $70 \mu \mathrm{m}$ ) are arranged in the verrucous surface in one direction, similar in appearance to strawberry seeds, and the structure is completely different from that observed in the congeners of the respective genera. Furthermore, according to Ohshima's (1913) illustrations and photographs, there is a possibility that the hook papilla of $T$. japonicus is not hollow, and it may have a completely different function from that of the two new species. An accurate knowledge of the structures of hook papillae in both the genera will be necessary in future studies.

Although DNA analysis and ossicle morphology clearly demonstrate that Scoliorhapis sesokoensis and Taeniogyrus verruculosus are separate species, they are not easily distinguished from each other based on the external appearance of their body shape and color. In external appearance, only the number of tentacle digits serves as a useful distinguishing feature in the adult forms. The number of tentacle digits of $T$. verruculosus is up to eight (seven digits in two paratype specimens, eight digits in the holotype), while $S$. sesokoensis, having up to six digits (in five type specimens, and more than 50 specimens of other materials, including young and adult). In the ossicle morphology, the most reliable distinguishing feature is the existence of wheels in the anterior dorsal skin of T. verruculosus. Another rough distinguishing feature is the size range of the shank length of sigmoid-hooks. The sigmoid-hooks of $T$. 
verruculosus are comparatively larger than that of S. sesokoensis in animals of similar body size. However, the size ranges of the shank length of sigmoid-hooks differ significantly among specimens and parts, and became smaller in small sized animals. Furthermore, in the apodid sea cucumbers, the apparent dimensions of the animals are easily reduced by autotomy and shrinkage. Therefore, it may be difficult to judge whether the sigmoid-hooks are large or small if there is not a complete specimen or anaesthetized specimen.

Based on our observations, wheel ossicles can reliably be detected in $T$. verruculosus. However, wheel ossicles were very sparse in all specimens, and nearly lacking in a paratype specimen (WMNH-INV-2016-205, in which we could detect one wheel only). If we ignore the presence of wheel ossicles, then the two new species closely resemble each other, and it would seem that they could be phylogenetically related. However, the results of DNA analysis demonstrated the dichotomy between the two species. However, we still question whether the amount of evolutionary distance between them justifies a level of difference that would occur between two genera, and further analyses we conduct will address this question.

\section{Acknowledgements}

We would like to thank Prof Susumu Ohtsuka of Takehara Marine Science Station of Hiroshima University for providing the equipment of DNA analysis, and Prof Hideyuki Yamashiro, Mr Yoshikatsu Nakano, and entire staff of Sesoko Station of Tropical Biosphere Research Center of University of the Ryukyus for many help during the survey in Sesoko Island. We also thank Dr Munenori Yamashita of Industrial Technology Center of Wakayama Prefecture for providing the equipment of SEM analysis, and $\mathrm{WMNH}$ for the invaluable assistance provided during this study, Mr Tohru Imaoka for his helpful advice, and $\mathrm{Mr}$ Yohei Yamana for assistance of collecting important specimens. We also thank two anonymous reviewers for reviewing the manuscript. This work was partially supported by the Research Institute of Marine Invertebrates (Tokyo) Grant 2015KO-1. Financial support for part of the microscopic observations was provided by Mr Shinichi Ikebe of the Supporters' Association of WMNH.

\section{References}

Altschul SF, Gish W, Miller W, Myers EW, Lipman DJ (1990) Basic local alignment search tool. Journal of Molecular Biology 215: 403-410.

Altschul SF, Madden TL, Schäffer AA, Zhang J, Zhang Z, Miller W, Lipman DJ (1997) Gapped BLAST and PSI-BLAST: a new generation of protein database search programs. Nucleic Acids Research 25: 3389-3402.

Arndt A, Marquez C, Lambert P, Smith MJ (1996) Molecular phylogeny of eastern Pacific sea cucumbers (Echinodermata:
Holothuroidea) based on mitochondrial DNA sequence. Molecular Phylogenetics and Evolution 6: 425-437.

Brandt JF (1835) Prodromus Descriptionis Animalium ab H. Mertensio Observatorum. Fasciculus I. Polypos, Acalephas Discophoras et Siphonophoras, nec non Echinodermata continens. Petropoli 1835: 201-275.

Clark HL (1907) The apodous holothurians. Smithsonian Contributions to Knowledge 35: 1-231.

Clark HL (1921) The Echinodermata fauna of Torres Strait: its composition and its origin. Carnegie Institution of Washington Publication 214: 1-233.

Clark HL (1946) The Echinoderm fauna of Australia: its composition and its origin. Carnegie Institution of Washington Publication 566: 1-567.

Folmer O, Black M, Hoeh W, Lutz R, Vrijenhoek R (1994) DNA primers for amplification of mitochondrial cytochrome oxidase subunit I from diverse metazoan invertebrates. Molecular Marine Biology and Biotechnology 3: 294-299.

Heding SG (1928) Synaptidae. Papers from Dr. Th. Mortensen's Pacific Expedition 1914-1916. No. 66. Videnskabelige Meddeleser fra Dansk naturhistorisk Forening i København 85: 105-323.

Heding SG (1931) Über die Synaptiden des Zoologischen Museums zu Hamburg. Zoologische Jahrbücher, Abteilung Systematik 61: 637-696.

Imaoka T (1995) Holothuroidea. In: Genshoku Kensaku Nihon Kaigan Doubutsu Zukan. Vol. II. [Guide to Seashore Animals of Japan with Color Pictures and Keys. Vol. II.] (ed Nishimura S). Hoikusha, Osaka, pp. 553-572. (In Japanese)

Kimura M (1980) A simple method for estimating evolutionary rate of base substitutions through comparative studies of nucleotide sequences. Journal of Molecular Evolution 16: 111120.

Ludwig H (1898) Holoturien. Ergebnisse der Hamburger Magalhaensischen Sammelreise (1892-93) 3: 1-98.

Marenzeller E (1882 [dated 1881]) Neue Holothurien von Japan and China. Verhandlungen der Zoologisch-Botanischen Gesellschaft in Wien 31: 121-140.

Mortensen T (1925) Papers from Dr. Th. Mortensen's Pacific Expedition 1914-16. XXIX. Echinoderms of New Zealand and the Auckland-Campbell Islands. III-V. Asteroidea, Holothurioidea and Crinoidea. Videnskabelige Meddeleser fra Dansk naturhistorisk Forening i København 79: 261-420.

Oguro C (1961) The fauna of Akkeshi Bay XXVI. Holothuroidea. Publications from the Akkeshi Marine Biological Station 11: $1-4$.

Ohshima H (1913) Synaptiden von Misaki. Dobutsugaku Zasshi 25: 253-262.

Ohshima H (1914) The Synaptidae of Japan. Annotationes Zoologicae Japonenses 8: 467-482.

Ohshima H (1915) Report on the holothurians collected by the United States Fisheries Steamer "Albatross" in the Northwestern Pacific during the summer of 1906. Proceedings of the United States National Museum 48: 213-291.

Ohshima H (1919) Holothuroidea in northwest Pacific, XX. Dobutsugaku Zasshi 31: 139-149. (In Japanese)

O’Loughlin PM, VandenSpiegel D (2010) A revision of Antarctic and Indo-Pacific apodid sea cucumbers (Echinodermata: Holo- 
thuroidea: Apodida). Memoirs of Museum Victoria 67: 61-95.

Östergren H (1898) Das System der Synaptiden. Öfversigt af Kongliga Vetenskaps-Akademiens Förhandlingar 55: 111-120.

Östergren H (1907) Zur Phylogenie und Systematik der Seewalzen. In: Särtryck ur Zoologiska studier tillãgnade (ed Tullberg T). Almquist et Wiksell, Uppsala, pp. 191-215.

Paulay G (2013) Taeniogyrus. Accessed through: World Register of Marine Species at http://www.marinespecies.org/aphia. php? $=$ taxdetails\&id=174000 (9 September 2015).

Paulay G (2015) Scoliorhapis Clark, 1946. Accessed through: World Register of Marine Species at http://www.marinespecies.org/aphia.php?p=taxdetails\&id=241391 (4 June 2016).

Semper C (1868) Holothurien. In: Reisen im Archipel der Philippinen, Teil 2, Wissenschaftliche Resultate (ed Semper C). C. W. Kreidel, Wiesbaden, pp. 1-288.

Smirnov AV (1998) On the classification of the apodid holothurians. In: Echinoderms: San Francisco (eds Mooi R, Telford M). Balkema, Rotterdam, pp. 517-522.
Smirnov AV (2012) System of the class Holothuroidea. Paleontological Journal 46: 793-832.

Solis-Marin FA, Komatsu M, Soliman T, Uchida K, Shimotani T, Nozaki M (2014) Scoliorhapis danthus, a new species of sea cucumber (Apodida: Chiridotidae) from the Sea of Japan. Proceedings of the Biological Society of Washington 127: 323-327.

Tamura K, Stecher G, Peterson D, Filipski A, Kumar S (2013) MEGA6: Molecular evolutionary genetics analysis version 6.0. Molecular Biology and Evolution 30: 2725-2729.

Théel H (1886) Report on the Holothurioidea dredged by H.M.S. Challenger during the years 1873-76. Part II. Report of the scientific results of the Voyage of H.M.S. Challenger during the years 1873-76. Zoology Part XXXIX. Volume 14: 1-290.

Utinomi H (1965) Holothuroidea. In: Shin Nihon Doubutsu Zukan. Vol. III. [New Illustrated Encyclopedia of the Fauna of Japan Vol. III] (eds Okada Y, Uchida S, Uchida T). Hokuryukan, Tokyo, pp. 82-99. (In Japanese) 